Central Washington University

ScholarWorks@CWU

All Faculty Scholarship for the College of the Sciences

5-27-1992

Tamm states in finite semiconductor superlattices: Influence of accumulation and depletion layers

Roger H. Yu

Follow this and additional works at: https://digitalcommons.cwu.edu/cotsfac

Part of the Atomic, Molecular and Optical Physics Commons, and the Quantum Physics Commons 


\title{
Tamm states in finite semiconductor superlattices: Influence of accumulation and depletion layers
}

\author{
R. H. Yu \\ Department of Physics, Central Washington University, Ellensburg, Washington 98926
}

(Received 27 May 1992)

\begin{abstract}
We discuss the electronic-level structure of finite semiconductor superlattices and the influence of accumulation and depletion regions on the surface localized states (Tamm states). Exact solutions of the Poisson and Schrödinger equations are obtained self-consistently, through use of a nonuniform jellium model. Both high-energy and low-energy Tamm states are found.
\end{abstract}

Recently, much interest has been devoted to the electronic subband structure and the dynamics of carriers near the surface of an otherwise uniform semiconductor. Two kinds of space-charge-related systems have been studied: (1) Depletion-layer systems, in which electrons are repelled away from the surface by charges trapped in surface states resulting from dangling bonds, defects, impurities, etc. The dynamics of electrons in such systems has been studied experimentally ${ }^{1}$ by means of electronenergy-loss spectroscopy (EELS), and theoretically ${ }^{2-4}$ by means of nonlocal dynamical response theory. (2) Accumulation-layer systems, in which electrons are attracted to the surface by the presence of an excess of opposite charges. Accumulation-layer systems can be created by exposing hydrogen atomic gas onto the surface [e.g., InAs, ${ }^{5,6} \mathrm{ZnO}$ (Refs. 7 and 8)], inducing donor levels which inject positive charges below the surface, bending the conduction band downward. An accumulation-layer system can also be achieved by applying an external electric field, or by contacting two materials with different band gaps (e.g., GaAs/ $\mathrm{Al}_{x} \mathrm{Ga}_{1-x} \mathrm{As}$ ). Experimental ${ }^{5-7}$ and theoretical ${ }^{2-6}$ studies of the electronic subband structure and the dynamics of electrons in accumulation layers have been carried out extensively.

In a finite superlattice, fabricated from layers of GaAs alternating with doped $\mathrm{Al}_{x} \mathrm{Ga}_{1-x}$ As layers, there also exist these same two kinds of layers, depletion layers and accumulation layers. A prime example of depletion layer in a semiconductor superlattice can be found in the quantum Hall effect (QHE) experiment ${ }^{9}$ performed by Stormer et al. The sample used in the experiment consisted of 30 layers of $188-\AA$ undoped $\mathrm{GaAs}$, alternating with 30 layers of $38-\AA$ silicon-doped $\mathrm{Al}_{x} \mathrm{Ga}_{1-x}$ As. Near the top layers of the superlattice, the depletion region was formed as a result of the pinning of the Fermi level below its position in intrinsic GaAs due to the midgap surface states. Stormer et al. also studied the miniband structure of the $\mathrm{GaAs} / \mathrm{Al}_{x} \mathrm{Ga}_{1-x}$ As superlattice using a simple KronigPenney model with cyclic boundary conditions. The discrepancies between the observed activation energy $\triangle$, defined as the difference between the Fermi energy and the closest energy level, and the calculated result was found. Later, Ulloa and Kirczenow ${ }^{10}$ performed a tightbinding calculation, in which the surface states associated with depletion layers were predicted, and agreement be- tween their calculations and the experimental results was achieved.

In this paper, we report results of the full selfconsistent calculations of the electronic energy-level structure of a finite modulation-doped superlattice. Much attention is concentrated on the influences of accumulation and depletion layers. An assumed surface charge $n_{s}$ is used to simulate trapped charges inside surface states that induce the accumulation layer for positive $n_{s}$ or the depletion layer for negative $n_{s}$. In contrast to our earlier work, ${ }^{6,8}$ where the ionized donor distribution was uniform and can be treated by the jellium model, a modulation-doped superlattice ionized donor distribution is no longer uniform. We have used a square-well function as the background donor distribution, which is similar to that of introducing atoms (although in one dimension) in jellium models.

Our model, described by the dashed lines in Figs. 1(a) and 1 (b), consists of the following sample parameters: $N$, the number of layers; nonuniform positive charge (donor) distribution $n_{D}(z)$, shown by the dashed line in Fig. 1(a); surface charge $n_{s}$ on both sides of our sample slab; square-well background potential $v_{B}(z)$, which is composed of the surface barrier $E_{s}$ and the interior barrier $E_{B}$, shown by the dashed line in Fig. 1(b). The conduction electrons inside the quantum wells are treated as free-electron gas in the plane parallel to the surface, and are characterized by an effective mass $m^{*}$. In the effective-mass approximation, the electron eigenenergies have the form

$$
E_{i}\left(\mathbf{k}_{\|}\right)=\frac{\hbar^{2} \mathbf{k}_{\|}^{2}}{2 m^{*}}+\varepsilon_{i},
$$

where the first term describes the energies of the free electron moving parallel to the surface, and the second term represents the energy levels of the quantized motion of electrons along the direction of growth. The singleparticle wave functions describing the quantized motion in the $z$ direction satisfy the Schrödinger equation

$$
\left[\frac{\hbar^{2}}{2 m^{*}} \frac{d^{2}}{d z^{2}}+v_{\mathrm{eff}}(z)\right] \phi_{i}(z)=\varepsilon_{i} \phi_{i}(z) .
$$

In the local-density approximation to the density- 
functional formalism, $v_{\text {eff }}(z)$ is the simple Hartree potential $v_{H}(z)$, which obeys the Poisson equation, supplemented by the background potential barriers shown by the dashed line in Fig. 1(b), and an exchange and correlation potential $v_{\mathrm{xc}}(z)$ of Hedin and Lundqvist. ${ }^{11}$ The Hartree potential $v_{H}(z)$ is the solution of the Poisson equation

$v_{H}(z)=-\frac{2 \pi e^{2}}{\varepsilon} \int_{0}^{L} d z^{\prime}\left|z-z^{\prime}\right|\left[n\left(z^{\prime}\right)-n_{D}\left(z^{\prime}\right)\right]$,

where $n(z)$ is the electron number density and $n_{D}(z)$ is the nonuniform doping density depicted by the dashed line in Fig. 1(a). Based on a guessed charge density $n(z)$, $v_{H}(z)$ is obtained from the integral in Eq. (3) to calculate $v_{\text {eff }}(z)$; subsequently, $\phi_{i}(z)$ is found by solving the Schrödinger equation, after which $n(z)$ is updated using the Fermi-Dirac distribution function. The Schrödinger and Poisson equations are solved iteratively until the self-consistency is reached. ${ }^{6,8}$ The Fermi energy is found by the condition of overall charge neutrality of the sample. 120 sine functions were used to expand the wave functions $\phi_{l}(z)$, and a similar expansion was applied to the effective potential $v_{\text {eff }}(z)$; finally, the Schrödinger
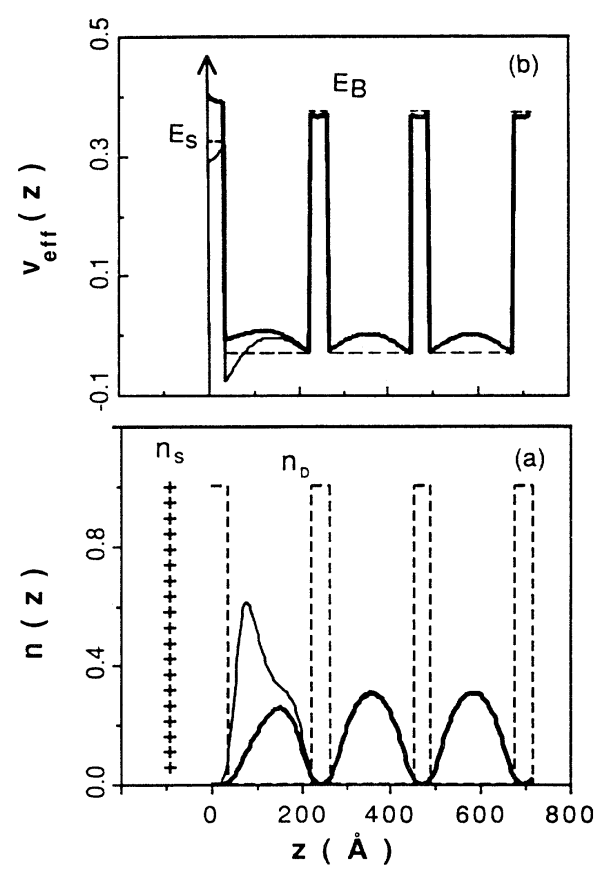

FIG. 1. (a) Self-consistent charge densities of the first three layers for the accumulation $\left(n_{s}=0.2 \times 10^{-4} \AA^{-2}\right)$ and depletion $\left(n_{s}=-0.4 \times 10^{-4} \AA^{-2}\right)$ layers are shown by the thin and thick solid lines, respectively. The background-doping-density profile is depicted by the dashed line. The following parameters were used in the calculation: barrier width $38 \AA$, well width $188 \AA$, number of layers $N=11, E_{B}=0.4 \mathrm{eV}, E_{s}=0.38 \mathrm{eV}$, $n_{D}=1.9 \times 10^{-6} \AA^{-3}, m^{*}=0.067 \mathrm{~m}$, and $\varepsilon=12.9$. (b) Selfconsistent potential $v_{\text {eff }}(z)$ of the first three layers for the accumulation (depletion) layer shown by the thin (thick) solid line. The background potential profile of the superlattice interior is shown by the dashed line. Note the difference between the surface barrier height $E_{s}$ and that of the interior, $E_{B}$. equation was transferred into a nonlinear matrix equation. $^{6,8}$

Figure 1 shows the self-consistent charge density and effective potential for the accumulation layer (thin solid line), $n_{s}=0.2 \times 10^{-4} \AA^{-2}$, and for the depletion layer (thick line), $n_{s}=-0.4 \times 10^{-2}$. The superlattice sample has 11 periods with $E_{B}=0.4 \mathrm{eV}$, and $E_{s}=0.38 \mathrm{eV}$, while the other parameters were chosen to match the experiment. ${ }^{9}$ The surface charges for both cases affect only the top layer because of the high potential barriers. The self-consistent charge-density profiles of interior layers for accumulation and depletion cases are strongly inhomogeneous. ${ }^{9}$ The electron charge densities at the doping sites are small but finite due to the weak tunneling effect; however, the charge densities at the centers of the wells are approximately 40 times larger than those at the centers of doped $\mathrm{Al}_{x} \mathrm{Ga}_{1-x}$ As layers, resulting in quasitwo-dimensional electron gases. In the outermost quantum well, the shift of the peak of the charge density is toward (away from) the surface for the accumulation (depletion) layer, originated in the fact that the positive (negative) surface charges attract (repel) the electrons. Because of the extra density of states at the surface, the weight of the charge density in the first well is different from that of the interior of the superlattice. Figure 1(b) shows the self-consistent effective potential $v_{\text {eff }}(z)$. For the modulation-doped $\mathrm{GaAs} / \mathrm{Al}_{x} \mathrm{Ga}_{1-x} \mathrm{As}$ superlattice, in which the $\mathrm{Al}_{x} \mathrm{Ga}_{1-x}$ As layers are doped with $\mathrm{Si},{ }^{9}$ electrons supplied by $\mathrm{Si}$ donors are transferred to the GaAs quantum wells and leave positive ions behind. For an individual interior well, the upward conduction-band bending shown in Fig. 1(b) is almost parabolic, indicating the linearity and symmetry of the electric field inside the well. Because of the small thickness of the barriers, the downward band bending in the barriers is visible but weak. For the depletion case, in the outermost layer the electrons are pushed inward into the superlattice as a result of the presence of the repulsive force on electrons; hence, the potential is raised near the surface. In contrast, for the accumulation case, in the outermost layer the bottom of the surface potential is depressed below its interior counterpart. ${ }^{12}$

The wave functions of occupied states for both accumulation and depletion layers are presented in Figs. 2(a) and 2(b), respectively, in the order of their corresponding energy levels. The most striking feature presented in Fig. 2 is the set of highly surface-localized states (also known as Tamm states). The justification for the existence of these surface-localized states is given by Tamm, ${ }^{13}$ who showed that if the Kronig-Penney square-well potential is terminated by a surface barrier, there will be discrete energy levels within the forbidden band gap. ${ }^{14}$ As we have already seen in the experiment ${ }^{9,15}$ and in the tightbinding calculations, ${ }^{10,16}$ the Tamm state exists above the miniband continuum for the depletion case, which is revealed in our results shown in Fig. 2(b). Because of the symmetry of our sample, the uppermost two modes, associated with both surfaces of the slab, are degenerate. Unlike the depletion layer, the accumulation layer supports a Tamm state (also twofold degenerate), which exists below the lowest miniband continuum as illustrated in 
Eigenfunctions
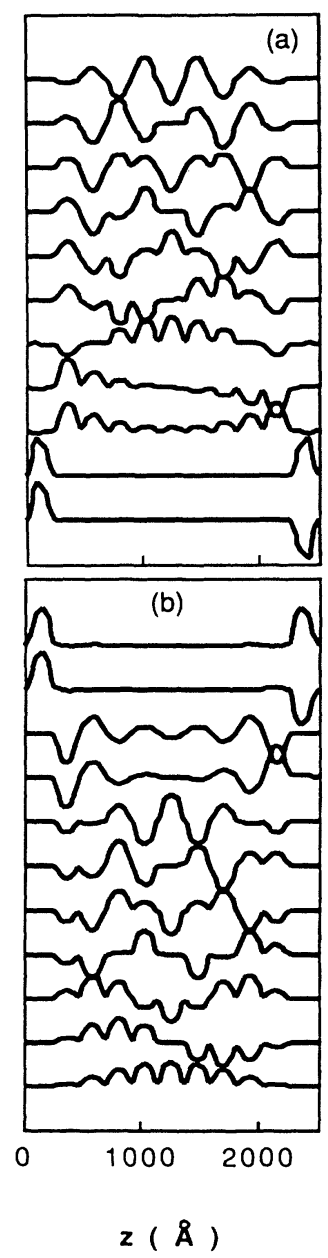

FIG. 2. (a) and (b) show the eigenfunctions of the lowest 11 occupied states for the accumulation and depletion layer, respectively, in the order of their corresponding energy levels.

Fig. 2(a). This unusual Tamm state is more heavily populated than that in the Tamm states above the miniband continuum at low temperature; therefore, the former "low-energy" Tamm state should play a more important role in electronic excitations. An experiment has been designated to study this "low-energy" Tamm state. ${ }^{15}$ Such a low-energy Tamm state can be obtained by lowering the potential barrier of the terminating layer, or by widening the outermost quantum well. The latter case had been investigated by Agullo-Rueda et al. ${ }^{17}$ By inspecting the delocalized electronic wave function, we find the evolution of the Bloch-like states corresponding to 11 quantum wells. In order to construct the dispersion relation, we evaluate the wave vector $k$ of the Bloch wave by finding the envelope function for each state. Our calculated dispersion relation of an 11-layer superlattice is slightly lower than that obtained by the Kronig-Penney model for an infinite superlattice.

To illustrate the effect of accumulation and depletion layers introduced by surface charges, we have depicted

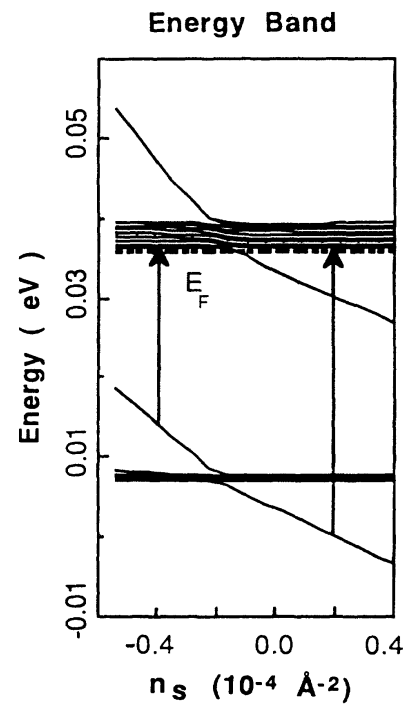

FIG. 3. Calculated energy levels as a function of surface charge $n_{s}$. The model parameters were chosen to be the same as given in Fig. 1.

eigenenergies as a function of surface charge $n_{s}$ in Fig. 3. As expected, two groups of energy levels form the two lowest minibands. For each miniband, there are two midgap-lying Tamm states which are surface localized. For an accumulation (depletion) layer, the energy level of the Tamm state is lower (higher) than the miniband continuum. Note the nearly linear behavior of the energy level of the Tamm state as a function of surface charge density $n_{s}$, and the anticrossing behavior of the Tamm state due to the interaction between it and the miniband continuum. If the terminating barrier is lower than the rest of the superlattice, the Tamm state exists below the miniband. ${ }^{14}$ In the sample used in our calculation, the surface barrier $E_{s}$ is lower than the interior barrier height $E_{B}$; therefore, the energy of the Tamm state shown in Fig. 3 is below the miniband for a slightly negative surface charge density. The arrows in Fig. 3 indicate values of surface charge density at which the self-consistent charge density and effective potential are calculated as shown in Fig. 1.

In conclusion, we have applied the self-consistent method to determine exactly the energy-level structure of a modulation-doped superlattice. The effect of accumulation and depletion layers has been studied. We have found a low-energy (high-energy) surface-localized state for the accumulation (depletion) layer. Our results also revealed the band structure of a bulk superlattice. Discrepancies between our results and those obtained by the Kronig-Penney model indicate the importance of a realistic model.

We would like to thank S. E. Ulloa and J. C. Hermansom for many useful discussions. This work was partially supported by the Faculty Research Fund of Central Washington University. 
${ }^{1}$ R. Malz and H. Luth, Phys. Rev. Lett. 46, 500 (1981); A. Ritz and H. Luth, ibid. 52, 1242 (1984); Z. J. Grey-Grychowski et al., Solid State Commun. 59, 703 (1986); L. H. du Bois et al., Phys. Rev. B 35, 9128 (1987); Y. Chen, S. Nannarone, J. Schaefer, J. C. Hermanson, J. Anderson, and G. Lapeyre, ibid. 39, 7653 (1989).

${ }^{2}$ D. H. Ehlers and D. L. Mills, Phys. Rev. B 34, 3939 (1986).

${ }^{3}$ D. H. Ehlers and D. L. Mills, Phys. Rev. B 36, 1051 (1987).

${ }^{4}$ S. R. Streight and D. L. Mills, Phys. Rev. B 40, 10488 (1989).

${ }^{5}$ Y. Chen, J. C. Hermanson, and G. Lapegre, Phys. Rev. B 39, 12682 (1989).

${ }^{6}$ R. H. Yu and J. C. Hermanson, Phys. Rev. B 40, 11851 (1989).

${ }^{7}$ A. Many, I. Wagner, A. Rosenthal, J. Gersten, and Y. Goldstein, Phys. Rev. Lett. 46, 1648 (1981); J. Gersten, I. Wagner, A. Rosenthal, Y. Goldstein, A. Many, and R. Kerby, Phys. Rev. B 29, 2458 (1984).
${ }^{8}$ R. H. Yu and J. C. Hermanson, Phys. Rev. B 41, 5991 (1990).

${ }^{9}$ H. L. Stormer, J. P. Eisenstein, A. C. Gossard, W. Wiegmann, and K. Baldwin, Phys. Rev. Lett. 56, 85 (1986).

${ }^{10}$ S. E. Ulloa and G. Kirczenow, Phys. Rev. Lett. 57, 2991 (1986).

${ }^{11}$ L. Hedin and B. I. Lundqvist, J. Phys. C 4, 2064 (1971).

${ }^{12}$ T. Ando, J. Phys. Soc. Jpn. 51, 3893 (1982).

${ }^{13}$ I. Tamm, Phys. Z. Sowjetunion 1, 733 (1932).

14J. P. McKelvey, Solid State and Semiconductor Physics (Harper \& Row, New York, 1966).

${ }^{15}$ H. Ohno, E. E. Mendez, J. A. Brum, J. M. Hong, F. AgulloRueda, L. L. Chang, and L. Esaki, Phys. Rev. Lett. 64, 2555 (1990).

16J. Zhang and S. E. Ulloa, Phys. Rev. B 38, 2063 (1988).

${ }^{17}$ F. Agullo-Rueda, E. E. Mendez, H. Ohno, and J. M. Hong, Phys. Rev. B 42, 1470 (1990). 